\title{
Influence of Pulse Duration on High-Precision Manufacturing of 3D Geometries
}

\author{
Simon Schwarz ${ }^{1}$, Stefan Rung ${ }^{1}$, Cemal Esen² and Ralf Hellmann ${ }^{1}$ \\ ${ }^{1}$ Applied Laser and Photonics Group, University of Applied Sciences Aschaffenburg, \\ Wuerzburger Strasse 45, 63743 Aschaffenburg, Germany \\ E-mail: Simon.Schwarz@h-ab.de \\ ${ }^{2}$ Applied Laser Technologies, Ruhr-University Bochum, Universitaetsstrasse 150, Bochum 44801, \\ Germany
}

\begin{abstract}
We report on the evaluation of different pulse durations between $230 \mathrm{fs}$ and $5 \mathrm{ps}$ on the layer-wise ablation of fused silica. In an initial step, the polished samples are roughened by scanning the laser across the surface and ablating hatches consisting of parallel lines, where chipping and cracking is present for longer pulse durations. The ablation behavior of the further layers showed two ablation regimens with respect to the laser fluence for all pulse durations. Furthermore, for longer pulses a more efficient ablation process is found. With increasing pulse duration, the surface roughness is increased, an undesirable effect for high-precision micromachining. To demonstrate our micromachining process, we defined 3D geometries, i.e., axicon, spherical and cylindrical lenses which are ablated with 230 fs by layer-wise removal of the silica. Laser scanning microscope images show the effective fabrication of the 3D objects. Cross-sections reveal excellent agreement between the designed and the experimentally fabricated geometries confirming our high-precision micromachining process.
\end{abstract}

DOI: $10.2961 /$ jlmn.2018.03.0025

Keywords: micromachining, femtosecond, pulse durations, ablation, 3D geometries

\section{Introduction}

Femtosecond laser micromachining is an innovative method to process transparent materials [1]. Compared to the use of longer laser pulses, femtosecond laser processing is well-known for its excellent machining quality due to an efficient energy deposition and minimum heat-affected zone [2-4]. During irradiation of dielectric materials with high intensity femtosecond laser pulses, electrons are excited from the valance to the conduction band via multi-photon absorption and avalanche ionization [5]. These free electrons are further excited and heated by the incident laser radiation, before the energy is transferred to the lattice causing ablation of the material [6]. This behavior offers great advantage for contact-less and thus gentle and wear-free optical fabrication with freely selectable geometry.

Optical elements with feature sizes on the micro-scale reveal potential in many fields of applications such as, e.g., optical communication, micro-opto-electro-mechanical systems, lab-on-chip devices and sensor applications [7-9]. Beside conventional manufacturing methods, numerous femtosecond laser-based processes have been reported for the fabrication of optical components. Different two-step processes including a femtosecond laser irradiation step followed by a wet etching step has been demonstrated by Tsai et al. [10] and Pan et al. [7] for the fabrication of microlenses. Guo et al. [11] used a femtosecond laser for the generation of microlenses with two-photon polymerization while Cheng et al. [12] used the femtosecond laser for the irradiation of photosensitive glass within a four-step process chain. Embedded micro-ball lenses have been demonstrated by Zheng et al.
[13], focusing a femtosecond laser inside PMMA. Choi et al. [9] and Delgado et al. [14] generated preforms with the femtosecond laser, while a second $\mathrm{CO}_{2}$ laser process is used as reshaping process. Within the latter process, the required geometry is introduced, accompanied by a surface polishing. Contrary to all these femtosecond laser based manufacturing processes, our process chain uses a femtosecond and a $\mathrm{CO}_{2}$ laser. The geometry of the optical components is generated by a high-precision femtosecond micro-machining process, followed by a $\mathrm{CO}_{2}$ laser polishing step [15]. Opposite to Ref. $[9 ; 14]$, the later step is applied to polish the surface without reshaping the afore fabricated structures.

Here, we report on the layer-wise micromachining of 3D geometries forming optical components with femtosecond laser pulses. In particular, for this process the influence of pulse duration between $230 \mathrm{fs}$ and 5 ps on the ablation of fused silica is investigated. For an initial roughening step of the samples, the laser is scanned across the surface, ablating hatches consisting of parallel lines. Chipping and cracking is found at the rim of the ablated craters for longer pulses, confirming the preferable use of femtosecond pulses for high-quality micromachining. For the following subsequent layers, two ablation regimens exists with respect to the applied fluence. Three exemplarily chosen optical components, namely an axicon, a spherical and a cylindrical lens, are defined to demonstrate the superior capabilities of the layerby-layer ablation process. Laser scanning microscope images show that the geometries of the constructed and laser generated 3D objects are in excellent agreement, confirming the high precision of the micromachining process. 


\section{Experimental}

In our experimental study, a $\mathrm{Yb}: \mathrm{KGW}$ ultrashort pulsed laser (Pharos, Light Conversion) having a wavelength of $1030 \mathrm{~nm}$ is used. The laser is equipped to a micromachining system including a galvo scanner (RTA AR800 2G+, Newson) with an $\mathrm{f}-\Theta$-lens having a focal length of $100 \mathrm{~mm}$, mounted on a motorized z-stage (PRO165, Aerotech). The laser system leads to a Gaussian focus diameter of $32 \mu \mathrm{m}\left(1 / \mathrm{e}^{2}\right)$ as being measured by a high resolution CCD camera (UI-1490SE-M-GL, IDS). An external attenuator is used to alter the pulse energy, while the pulse duration $\tau$ is varied between $230 \mathrm{fs}$ and $5 \mathrm{ps}$ (FWHM) in our study with a pulse repetition rate of $50 \mathrm{kHz}$. Fused silica (GVB solutions in glass) is chosen as a prominent candidate in optics fabrication. To analyze the ablated structures in detail, a scanning electron microscope (Phenom ProX, Phenom-World) and a laser scanning microscope (VK-X210, Keyence) are used. The ablated step height $h$ and the surface roughness $S_{a}$ is measured in areas of $100 \mu \mathrm{m} \times 200 \mu \mathrm{m}$.

\section{Results and discussion}

In the micromachining process under study here, the 3D predefined geometry is ablated layer-by-layer, while for each layer a hatch consisting of parallel lines is used. To ensure a homogenous fluence input throughout the ablation process, a theoretical approach is used to determine the required pulse distance PD (distance between two adjacent laser pulse centers in $\mathrm{x}$ - and $\mathrm{y}$-direction) and thus the line distance within the hatch and the corresponding scanning speed. Therefore, the accumulated fluence $\Phi_{\text {acc }}$ for the overlapping pulses is calculated with equation 1 , where $\omega_{0}$ describes the beam radius, $\Phi_{0}$ the single pulse peak fluence, $x_{i}$ and $y_{i}$ the relative central positions of the $i_{\text {th }}$ and the $j_{\text {th }}$ pulse while $i$ and $\mathrm{j}$ are integer numbers [16].

$$
\Phi_{a c c}(\mathrm{x}, \mathrm{y})=\sum_{i=-\infty}^{\infty} \sum_{j=-\infty}^{\infty} \Phi_{0} e^{-\frac{2\left(\left(x-x_{i}\right)^{2}+\left(y+y_{i}\right)^{2}\right)}{\omega_{0}^{2}}}
$$

Figure 1 shows the accumulated fluence for different pulse distances, i.e., $12 \mu \mathrm{m}, 14 \mu \mathrm{m}, 16 \mu \mathrm{m}$ and $18 \mu \mathrm{m}$. The fluence is calculated for $10 \times 10$ pulses and exemplarily $\Phi_{0}=1 \mathrm{~J} / \mathrm{cm}^{2}$ while the beam radius is set to $16 \mu \mathrm{m}$ as in our experiments. For greater PD, the individual laser pulses are clearly visible and increasingly disappear for smaller PD while the accumulated fluence increases. At $\mathrm{PD}=12 \mu \mathrm{m}$ the accumulated fluence reaches a level of $2.8 \mathrm{~J} / \mathrm{cm}^{2}$ (figure 1) while a homogenous fluence distribution exists. Therefore,

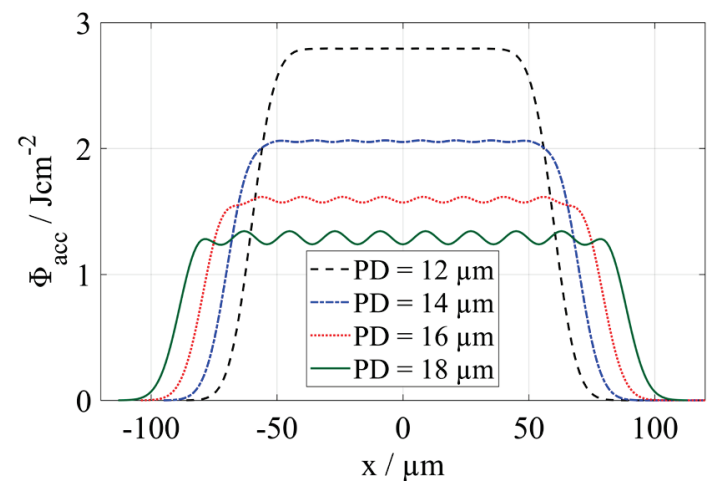

Fig. 1 Accumulated fluence for $10 \times 10$ pulses for pulse distances of $12 \mu \mathrm{m}, 14 \mu \mathrm{m}, 16 \mu \mathrm{m}$ and $18 \mu \mathrm{m}$.

Table 1 Results of the initial roughened layer for the different pulse durations.

\begin{tabular}{cccc}
\hline$\tau[\mathrm{fs}]$ & $\Phi\left[\mathrm{J} / \mathrm{cm}^{2}\right]$ & $\mathrm{h}[\mu \mathrm{m}]$ & $\mathrm{S}_{\mathrm{a}}[\mu \mathrm{m}]$ \\
\hline 230 & 2.82 & 1.451 & 0.367 \\
500 & 3.66 & 2.711 & 0.434 \\
1000 & 3.87 & 2.767 & 0.507 \\
2500 & 3.87 & 9.947 & 1.013 \\
5000 & 5.14 & 12.834 & 0.948 \\
\hline
\end{tabular}

we choose this pulse distance as preferential parameter for our laser ablation, resulting in a scanning speed of $600 \mathrm{~mm} / \mathrm{s}$ and a distance between the several lines within the hatch of $12 \mu \mathrm{m}$. To achieve a well controllable ablation process, initially the polished fused silica specimens have to be roughened prior to the $3 \mathrm{D}$ structuring to guarantee a comparable surface morphology and thus a comparable absorption behavior of the laser pulses. Therefore, we ablated hatches having half the pulse distance as defined in our theoretical approach for a homogeneous accumulated fluence of the overlapping pulses (see figure 1). Hence, a line distance of $6 \mu \mathrm{m}$ and a scanning speed of $300 \mathrm{~mm} / \mathrm{s}$ is chosen, resulting in a pulse distance of $6 \mu \mathrm{m}$ in $\mathrm{x}$ - and $\mathrm{y}$-direction. The single pulse fluence $\Phi$ is varied for the individual hatches to determine the fluence required for a complete roughening of the surface for the different pulse durations. We found an increasing fluence from $2.82 \mathrm{~J} / \mathrm{cm}^{2}$ for $230 \mathrm{fs}$ up to $5.14 \mathrm{~J} / \mathrm{cm}^{2}$ for $5 \mathrm{ps}$ for the initial roughening, taken from the first completely roughened fields.

Figure 2 shows scanning electron microscopy (SEM)

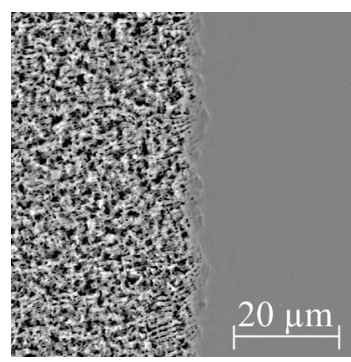

(a)

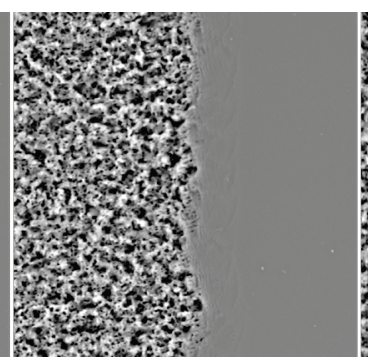

(b)

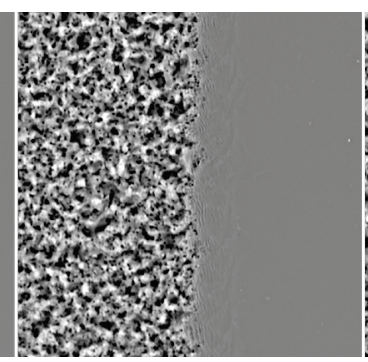

(c)

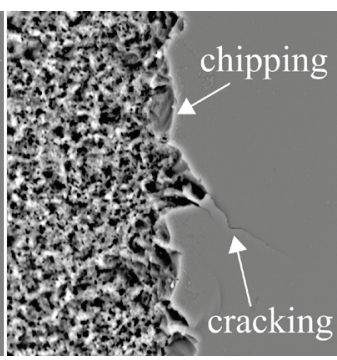

(d)

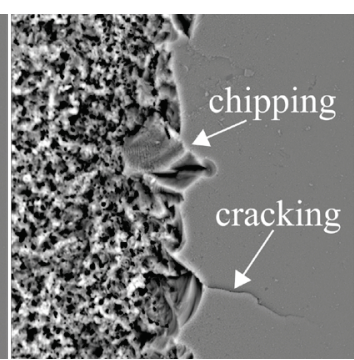

(e)

Fig. 2 SEM images of the initial roughened layers (a) $\tau=230 \mathrm{fs}, \Phi=2.82 \mathrm{~J} / \mathrm{cm}^{2}$, (b) $\tau=500 \mathrm{fs}, \Phi=3.66 \mathrm{~J} / \mathrm{cm}^{2},(\mathrm{c}) \tau=1 \mathrm{ps}$, $\Phi=3.87 \mathrm{~J} / \mathrm{cm}^{2}$, (d) $\tau=2.5 \mathrm{ps}, \Phi=3.87 \mathrm{~J} / \mathrm{cm}^{2}$, (e) $\tau=5 \mathrm{ps}, \Phi=5.14 \mathrm{~J} / \mathrm{cm}^{2}$. 


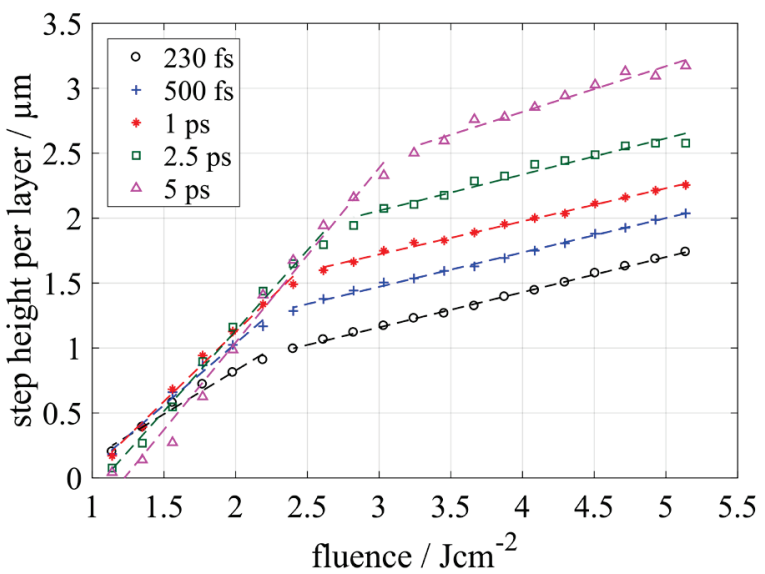

(a)

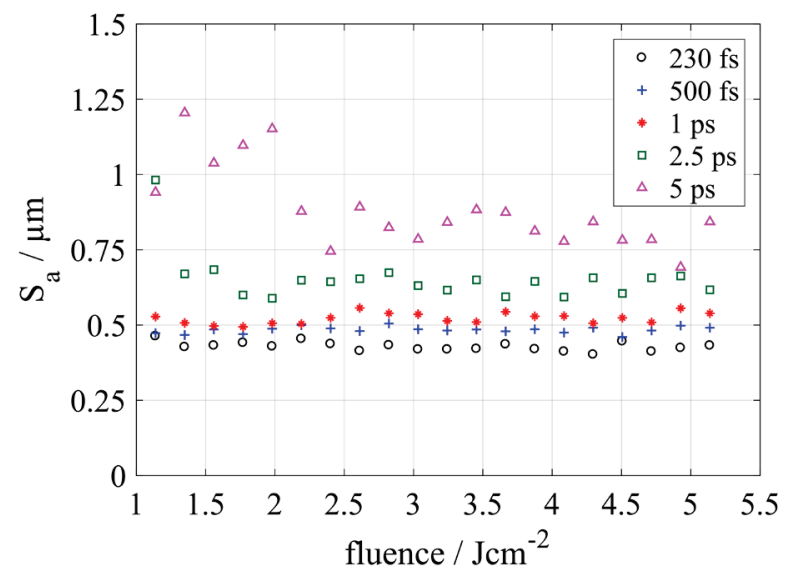

(b)

Fig. 3 Step height per layer (a) and surface roughness (b) of the ablated areas for the different pulse durations between $230 \mathrm{fs}$ and 5 ps.

images of roughened areas for different pulse durations and the lowest fluences leading to a complete roughening. Obviously, roughening with pulse lengths of 2.5 ps and 5 ps reveals chipping and cracking at the rim of the ablated areas (figure 2 (d) and (e)). These defects can clearly be assigned to the pulse length, since for the same fluence applied for $1 \mathrm{ps}$ and $2.5 \mathrm{ps}$ pulses, yet shorter pulse length of $1 \mathrm{ps}$ (figure 2 (c)), they completely disappear. The ablated step height $h$ as well as the surface roughness $S_{a}$ increase with increasing pulse duration, as summarized in table 1 . Please note, in addition to the increasing pulse duration, the fluence is also increased (except for $1 \mathrm{ps}$ and $2.5 \mathrm{ps}$ ). For the initial roughening using a pulse length of 5 ps, we find a step height of $12.83 \mu \mathrm{m}$, which is about a factor of 9 higher than the corresponding height for 230 fs. Overall, we can identify the shortest pulse length of $230 \mathrm{fs}$ as being superior for the ablation process, as it leads to smallest values for the required fluence, achieved step height as well as surface roughness and disappearing chipping and cracking.

After the initial surface roughening, the morphology and absorption behavior of fused silica has changed. To evaluate the layer-by-layer ablation process in detail, first the surface is roughened with the parameters determined in table 1 for the individual pulse durations, followed by the ablation of hatches with fluences between $1.14 \mathrm{~J} / \mathrm{cm}^{2}$ and $5.14 \mathrm{~J} / \mathrm{cm}^{2}$. For this ablation the PD is set to $12 \mu \mathrm{m}$ as found for a homogenous $\Phi_{\text {acc }}$ within our theoretical approach (see figure 1), resulting in a scanning speed of $600 \mathrm{~mm} / \mathrm{s}$ and a line distance within the several hatches of $12 \mu \mathrm{m}$. For a more accurate interpretation of the results, 10 layers are ablated after the roughening. In accordance to the later ablation of $3 \mathrm{D}$ geometries, after each of these 10 layers, the hatch is rotated by $100^{\circ}$ to ensure a more homogeneous ablation process. Figure 3 (a) summarizes the ablated step height per layer (initial roughening layer excluded) for the different pulse durations. Apparently, two regimes (lower and higher fluence regime) are found. In both regimes, the ablated step height increases linear with the applied fluence while for the higher fluence regime, the regression lines are almost parallel, whereas the slope for the lines in the lower fluence regime increase with increasing pulse duration. Furthermore, we find a more efficient ablation for longer pulse durations in
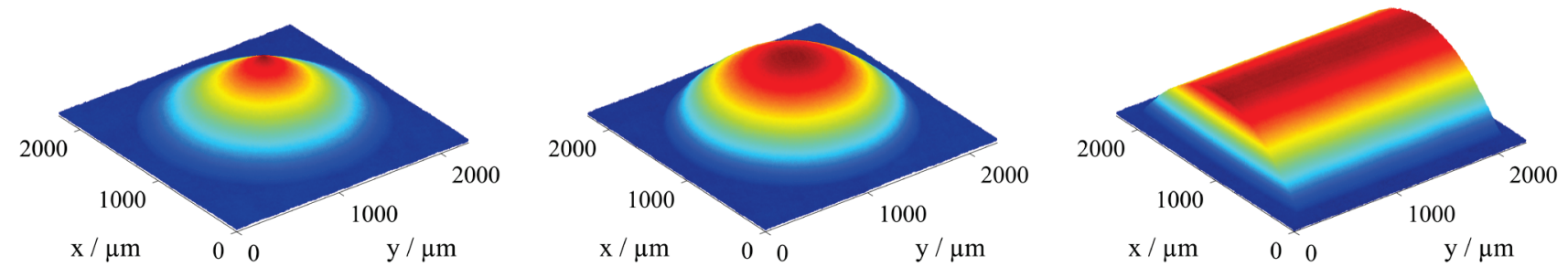

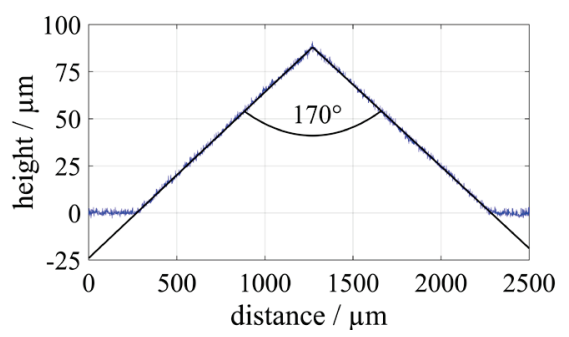

(a)

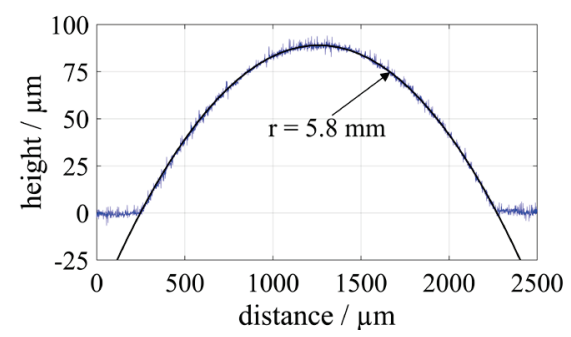

(b)

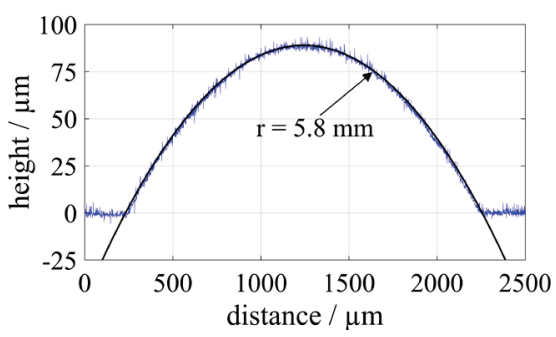

(c)

Fig. 4 3D- and 2D-LSM images of the fabricated geometries (a) axicon, (b) spherical lens and (c) cylindrical lens. Black lines show the cone angle and the radius of the stipulated geometries. 
the higher fluence regimen i.e. greater step height per layer at the same fluence level. Figure 3 (b) shows the surface roughness of the ablated areas. Obviously, the surface roughness increases with increasing pulse duration, while the lowest values of $S_{a}$ can be found for 230 fs being underneath $0.5 \mu \mathrm{m}$ over the entire fluence range.

After an in-depth evaluation of the ablation process of fused silica for different pulse durations, we found for an initial roughening the shortest pulse duration of $230 \mathrm{fs}$ is preferable. For the further layers, longer pulse durations proved to be more efficient due to the higher ablation rates. Against the background of high-precision layer-by-layer fabrication of $3 \mathrm{D}$ geometries for optical components, the $230 \mathrm{fs}$ fits the requirements best due to the lowest surface roughness.

To demonstrate the capabilities of the proposed highprecision 3D ablation process, we designed three objects, forming the basic geometry of the optical elements axicon, spherical and cylindrical lens. The structures are designed to have diameters (axicon, spherical lens) or an edge length (cylindrical lens) of $2 \mathrm{~mm}$ and a height of $87 \mu \mathrm{m}$, resulting in a cone angle for the axicon of $170^{\circ}$ and a radius for the spherical and cylindrical structures of $5.8 \mathrm{~mm}$. Please note, the different structures are designed as negative to remove the surrounding material, remaining the desired geometry. Based on the above described result, for the ablation process the shortest pulse duration of $230 \mathrm{fs}$ is chosen while the initial layer is roughened with $\Phi=2.82 \mathrm{~J} / \mathrm{cm}^{2}$ and $\mathrm{PD}=6 \mu \mathrm{m}$. For the layer-wise ablation of the fused silica, we use $\Phi=2.42 \mathrm{~J} / \mathrm{cm}^{2}$ and $\mathrm{PD}=12 \mu \mathrm{m}$, while the focus is stepwise readjusted with the motorized z-stage after removing an individual layer. The fluence is chosen as to remove $1 \mu \mathrm{m}$ per layer. Figure 4 shows laser scanning microscope (LSM) images of the $3 \mathrm{D}$ structures and cross-sections for the three elements. Apparently, the femtosecond laser ablation process defines precisely the intended structures. The cross-sections reveal excellent agreement between the constructed and the experimental fabricated geometry. Thus, the axicon has a cone angle of $170^{\circ}$ while the spherical and the cylindrical lenses have a radius of $5.8 \mathrm{~mm}$. Again, we emphasize the excellent contour accuracy highlighting the high-precision of the layer-by-layer micromachining process.

For the use of our manufactured 3D optical elements, a second polishing step is mandatory to reduce the resulting surface roughness. In previous publications [15] we have demonstrated the feasibility of a $\mathrm{CO}_{2}$ laser for polishing the femtosecond laser generated structures. In this paper, we only focussed on the laser ablation process with respect to different pulse durations.

\section{Conclusion}

We have demonstrated the precise ablation of 3D geometries in fused silica using a femtosecond laser. Pulse durations between 230 fs and 5 ps are investigated in two steps (initial material roughening and further ablation). For the initial roughening, the laser is scanned across the surface to ablate hatches consisting of parallel lines. Chipping and cracking is found for longer pulse durations while for the shorter durations the ablation is more gentle. For further layers, all pulse durations reveal two ablation regimens by varying the fluence while the ablation process is more efficient for longer pulse durations. For the fabrication of $3 \mathrm{D}$ geometries, the shortest pulse duration of $230 \mathrm{fs}$ is chosen due to the lowest surface roughness. The manufacturing of axicon, spherical and cylindrical preforms reveal excellent agreement between the constructed and experimentally ablated objects, highlighting our high-precision ablation process.

\section{References}

[1] R. R. Gattass and E. Mazur: Nature Photon., 2, (2008) 219.

[2] B. N. Chichkov, C. Momma, S. Nolte, F. von Alvensleben and A. Tünnermann: Appl. Phys. A, 63, (1996) 109.

[3] E. Bulushev, V. Bessmeltsev, A. Dostovalov, N. Goloshevsky and A. Wolf: Opt. Lasers Eng., 79, (2016) 39.

[4] S. Ameer-Beg, W. Perrie, S. Rathbone, J. Wright, W. Weaver and H. Champoux: Appl. Surf. Sci., 127-129, (1998) 875.

[5] B. C. Stuart, M. D. Feit, A. M. Rubenchik, B. W. Shore and M. D. Perry: Phys. Rev. Lett., 74, (1995) 2248.

[6] M. Lenzner, J. Krüger, S. Sartania, Z. Cheng, C. Spielmann, G. Mourou, W. Kautek and F. Krausz: Phys. Rev. Lett., 80, (1998) 4076.

[7] A. Pan, B. Gao, T. Chen, J. Si, C. Li, F. Chen and X. Hou: Opt. Express, 22, (2014) 15245.

[8] C. H. Lin, L. Jiang, Y. H. Chai, H. Xiao, S. J. Chen and H. L. Tsai: Appl. Phys. A, 97, (2009) 751.

[9] H.-K. Choi, J. Ryu, C. Kim, Y.-C. Noh, I.-B. Sohn and J.-T. Kim: J. Laser Micro/Nanoeng., 11, (2016) 341.

[10] H.-Y. Tsai, S.-W. Luo and T.-L. Chang: CIRP Ann., 64, (2015) 205.

[11]R. Guo, S. Xiao, X. Zhai, J. Li, A. Xia and W. Huang: Opt. Express, 14, (2006) 810.

[12] Y. Cheng, H. L. Tsai, K. Sugioka and K. Midorikawa: Appl. Phys. A, 85, (2006) 11.

[13] C. Zheng, A. Hu, R. Li, D. Bridges and T. Chen: Opt. Express, 23, (2015) 17584.

[14] T. Delgado, D. Nieto and M. T. Flores-Arias: Opt. Lasers Eng., 86, (2016) 29.

[15] S. Schwarz and R. Hellmann: J. Laser Micro/Nanoeng., 12, (2017) 76 .

[16] D. F. Farson, H. W. Choi, B. Zimmerman, J. K. Steach, J. J. Chalmers, S. V. Olesik and L. J. Lee: J. Micromech. Microeng., 18, (2008) 035020.

(Received: June 20, 2018, Accepted: November 18, 2018) 